\title{
CUIDADO INTERPROFISSIONAL À CRIANÇA E AO ADOLESCENTE: REVISÃO DE ESCOPO
}

\section{INTERPROFESSIONAL CARE FOR CHILDREN AND ADOLESCENTS: SCOPING REVIEW}

\author{
Caroline Lima Fonseca* \\ ORCID: https://orcid.org/0000-0001-5974-4328 \\ Larissa de Almeida Costa** \\ ORCID: https://orcid.org/0000-0001-7751-1931 \\ Bruna Hinnah Borges Martins de Freitas*** \\ ORCID: https://orcid.org/0000-0002-6652-593X \\ Lidiane Cristina da Silva Alencastro**** \\ ORCID: https://orcid.org/0000-0003-3005-415X \\ Marcus Vinícius Mizoguchi***** \\ ORCID: https://orcid.org/0000-0002-7680-4393
}

\section{Resumo}

O objetivo deste estudo foi mapear as evidências disponíveis acerca do cuidado interprofissional à criança e ao adolescente. Trata-se de uma revisão de escopo desenvolvida por extensionistas do PET-Saúde/Interprofissionalidade. Selecionaram-se estudos primários publicados entre 2009 e 2019 que abordavam o cuidado interprofissional à criança e ao adolescente. Foram incluídos 35 artigos. A partir de seus conteúdos, foram estabelecidas três categorias: a importância da educação interprofissional para o cuidado colaborativo em pediatria; potencialidades e desafios da colaboração interprofissional em pediatria; e as implicações do cuidado interprofissional à criança e ao adolescente. Esta revisão resultou no mapeamento do cuidado interprofissional às crianças e aos adolescentes no contexto mundial, revelando o potencial da educação interprofissional e do cuidado colaborativo no campo da pediatria. Além disso, esses achados poderão subsidiar mudanças na educação e nas práticas de saúde à luz da interprofissionalidade em prol de melhorias no cuidado infantojuvenil.

Palavras-chave: Cuidado de saúde; Relações interprofissionais; Educação interprofissional; Saúde da criança; Saúde do adolescente.

\begin{abstract}
The aim of this study was to map the available evidence about interprofessional care for children and adolescents. It is a scoping review developed by the extensionists from "PET-Saúde/Interprofessionalidade". We selected primary studies published between 2009 and 2019 that addressed interprofessional care for children and adolescents. We included 35 articles. Based on its contents, three categories were established: the importance of interprofessional education for collaborative care in pediatrics; potentials and challenges of interprofessional collaboration in pediatrics; and the implications of interprofessional care for children and adolescents. This review resulted in mapping the interprofessional care for children and adolescents in the global context, revealing the potential of interprofessional education and collaborative care in the field of pediatrics. In addition, the findings may support changes in education and health practices in the light of interprofessionality, providing improvements in infant and juvenile care.
\end{abstract}

Keywords: Health Care; Interprofessional Relations; Interprofessional Education; Child health; Adolescent Health.

Data recebimento: $28 / 01 / 2021$

Data de aceite: 04/05/2021
* Aluna de Graduação da Universidade Federal de Mato Grosso (UFMT), Cuiabá - MT, Brasil. E-mail: carolinefonseca99@gmail.com

** Aluna de Graduação da Universidade Federal de Mato Grosso (UFMT), Cuiabá - MT, Brasil. E-mail: larissacosta1212@gmail.com

*** Professora e aluna de Doutorado da Universidade Federal de Mato Grosso (UFMT), Cuiabá - MT, Brasil. E-mail: bruhinnah@gmail.com

**** Professora da Universidade Federal de Mato Grosso (UFMT), Cuiabá - MT, Brasil. E-mail: lidiane.alencastro@gmail.com

***** Professor da Universidade Federal de Mato Grosso (UFMT), Cuiabá - MT, Brasil. E-mail: marcusmaringa@gmail.com 


\section{Introdução}

O cuidado é um construto evidenciado em diversos campos de estudo na contemporaneidade. Embora cada campo disciplinar tenha sua origem e abordagem própria, a base do cuidado se configura por meio do entendimento das relações humanas a partir das experiências cotidianas (AYRES, 2017). O sentido norteador da integralidade deste cuidado está centrado na não fragmentação da atenção em saúde que, por sua vez, deve respeitar os sujeitos, seus direitos e suas necessidades, além de buscar interações e articulações entre os diferentes saberes, profissionais, serviços e setores voltados à saúde (KALICHAMAN; AYRES, 2016).

No que tange ao cuidado à criança e ao adolescente, torna-se necessário uma atenção à saúde voltada às suas necessidades e vulnerabilidades específicas, respeitando seus direitos e os contextos em que vivem. Este deve ser direcionado por iniciativas para assistência e controle de doenças, prevenção de enfermidades e agravos, pautando-se na promoção e nos determinantes da saúde. Destaca-se que este olhar deve permear todos os níveis de serviços de saúde e setores envolvidos no cuidado a este grupo etário, seja na atenção primária, secundária, terciária, ou mesmo nas escolas.

Nesta perspectiva, o trabalho colaborativo em rede é essencial, com a implementação de estratégias e alternativas mais efetivas e criativas para a articulação entre os sujeitos envolvidos, as instituições e os serviços. Tais ações possibilitam o rompimento de barreiras do desconhecimento sobre o modo de cuidado colaborativo, dos problemas relacionados ao processo de trabalho e da desarticulação da rede (TEIXEIRA; COUTO; DELGADO, 2017).

A colaboração interprofissional (CIP) desponta-se como uma das melhores formas de enfrentamento dos desafios altamente complexos do cuidado integral em pediatria (FARIAS et al., 2017). A CIP corresponde a prática profissional em que se desenvolve o trabalho interprofissional em saúde, articulando diferentes campos de práticas e fortalecendo a centralidade na pessoa, família e comunidade, e suas necessidades na dinâmica da produção dos serviços de saúde (MORGAN; PULLON; MCKINLAY, 2015). Anseia-se pela participação do usuário, família e comunidade na busca de "cuidar com as pessoas", ao invés de "cuidar para as pessoas" (DOMAJNKO et al., 2015).

Nos últimos anos, constatou-se um esforço crescente para a incorporação da CIP em todo o mundo. A Organização Pan-Americana da Saúde (OPAS) e a Organização Mundial da Saúde (OMS) têm estimulado esta proposta a partir de diversas iniciativas para que os Estados Membros apoiem e viabilizem a educação interprofissional (EIP) e a CIP através de políticas de educação e saúde (SILVA; CASSIANI; FREIRE FILHO, 2018). A EIP possibilita o desenvolvimento de competências para o trabalho interprofissional (CASANOVA; BATISTA; MORENO, 2018). Os pesquisadores destacam que este formato de aprendizagem contribui para o entendimento da importância da atuação em conjunto, potencializando os pontos fortes e as suas habilidades com uma visão mais ampla da saúde e do bem-estar da pessoa.

Este modelo gera a qualificação dos processos de trabalho colaborativos em saúde (MIGUEL et al., 2016). Portanto, salienta-se que a CIP tem grande potencial para dar sustentação a ações integrais e mais resolutivas, sobretudo por centralizar-se nas necessidades de saúde do cliente pediátrico e família, as quais são complexas e exigem a conexão de vários saberes. Apesar deste entendimento sobre a CIP, faltam estudos sobre as evidências disponíveis neste 
campo de conhecimento. Portanto, o objetivo do presente estudo foi mapear as evidências disponíveis acerca do cuidado interprofissional à criança e ao adolescente.

\section{Método \\ Desenho da pesquisa}

Trata-se de uma revisão de escopo, a qual visa a obtenção das evidências disponíveis de forma abrangente sobre determinada temática por meio do mapeando da literatura existente. Dessa forma, é possível identificar lacunas na base do conhecimento pesquisada (PETERS et al., 2020).

Trata-se de uma pesquisa desenvolvida por membros do projeto de extensão InterSaúde na Escola, subgrupo do "Programa Educação pelo Trabalho para a Saúde - Interprofissionalidade" (PET-Saúde/Interprofissionalidade) da Universidade Federal de Mato Grosso (UFMT). Este grupo atua no apoio aos processos de mudanças curriculares, alinhadas às Diretrizes Curriculares Nacionais para os cursos de graduação na área da saúde, considerando-se estratégias ligadas aos princípios da interprofissionalidade, interdisciplinaridade e intersetorialidade. $\mathrm{Na}$ lógica da formação dos profissionais e na dinâmica da produção do cuidado em saúde, e, ainda, na qualificação dos processos de integração ensino-serviço-comunidade, isso se dá de forma articulada entre o Sistema Único de Saúde (SUS) e a UFMT, de modo a se promover a Educação Interprofissional (EIP) e Práticas Colaborativas em Saúde da criança e adolescente.

A revisão seguiu as seguintes etapas: 1) definir e alinhar o (s) objetivo(s) e a pergunta; 2) definir os critérios de inclusão de acordo com o(s) objetivo(s) e a pergunta norteadora; 3) descrever a abordagem planejada para busca de evidências, seleção, extração de dados e apresentação das evidências; 4) buscar por evidências; 5) selecionar as evidências; 6) extrair as evidências; 7) analisar as evidências; 8) apresentar os resultados e 9) resumir as evidências em relação ao objetivo da revisão, refletir sobre as conclusões e observar quaisquer implicações dos resultados. O relatório desta revisão foi norteado pelas recomendações das diretrizes do Preferred Reporting Items for Systematic Reviews and Meta-Analyses Extension for Scoping Reviews (PRISMA-ScR) (PETERS et al., 2020).

\section{Etapa 1-5: Questão de pesquisa, critérios de inclusão, estratégia de busca e seleção dos estudos}

A pergunta de pesquisa foi elaborada por meio da estratégia PCC, que preconiza como elementos fundamentais o mnemônico: P - População; C- Conceito e C- Contexto (PETERS et al., 2020). Foram definidos os elementos: $\mathrm{P}$ (crianças e adolescentes); $\mathrm{C}$ (interprofissionalidade) e C (cuidados de saúde). A partir dessa definição, foi elaborada a seguinte pergunta de pesquisa: "Quais as evidências disponíveis sobre o cuidado interprofissional à criança e ao adolescente?".

Para esta revisão, as seguintes bases de dados foram definidas: National Library of Medicine (MEDLINE/PUBMED), Current Nursing and Allied Health Literature (CINAHL), SCOPUS, Web of Science (WOS) e Literatura Latino-americana e do Caribe em Ciências da Saúde (LILACS). Ressalta-se que essas bases foram selecionadas por serem as principais na área da saúde e terem o acesso via Portal de Periódicos da Coordenação de Aperfeiçoamento 
de Pessoal de Nível Superior (CAPES), a partir da identificação por meio da Comunidade Acadêmica Federada (CAFe) no Brasil.

Sequencialmente, os descritores controlados [Descritores em Ciência da Saúde (DeCS), Medical Subject Headings (MeSH) e CINAHL Headings] e não controlados (palavras-chave) foram elencados. Eles foram sintetizados conforme a estratégia PCC e suas combinações foram usadas para construir as estratégias: P: "adolescent" OR "child" AND C: "interprofessional relations" OR "interprofessionality" OR "collaborative practice" AND C "child care" OR "health care". A busca nas bases foi realizada no período de agosto a setembro de 2019 por dois revisores de forma independente.

Então, os artigos foram pré-selecionados a partir da leitura dos títulos e resumos nas bases. A amostra final foi alcançada com base na leitura dos artigos na íntegra, a partir dos critérios de elegibilidade.

Foram incluídos os estudos primários, nos idiomas inglês, espanhol ou português, e publicados entre 2009 e 2019. Optou-se por essa temporalidade porque, segundo o Global Forum on Innovation in Health Professional Education (2013), foi apenas nos últimos anos que a EIP passou a ser mais explorada nas pesquisas, políticas e atividades regulatórias internacionalmente. Foram excluídas as publicações não encontradas na íntegra, as que não responderam à questão de pesquisa e as duplicadas.

O fluxo de seleção dos artigos pode ser visualizado na Figura 1.

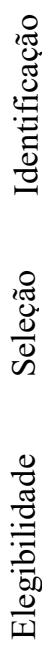

Figura 1 - Diagrama de fluxo da seleção dos artigos.

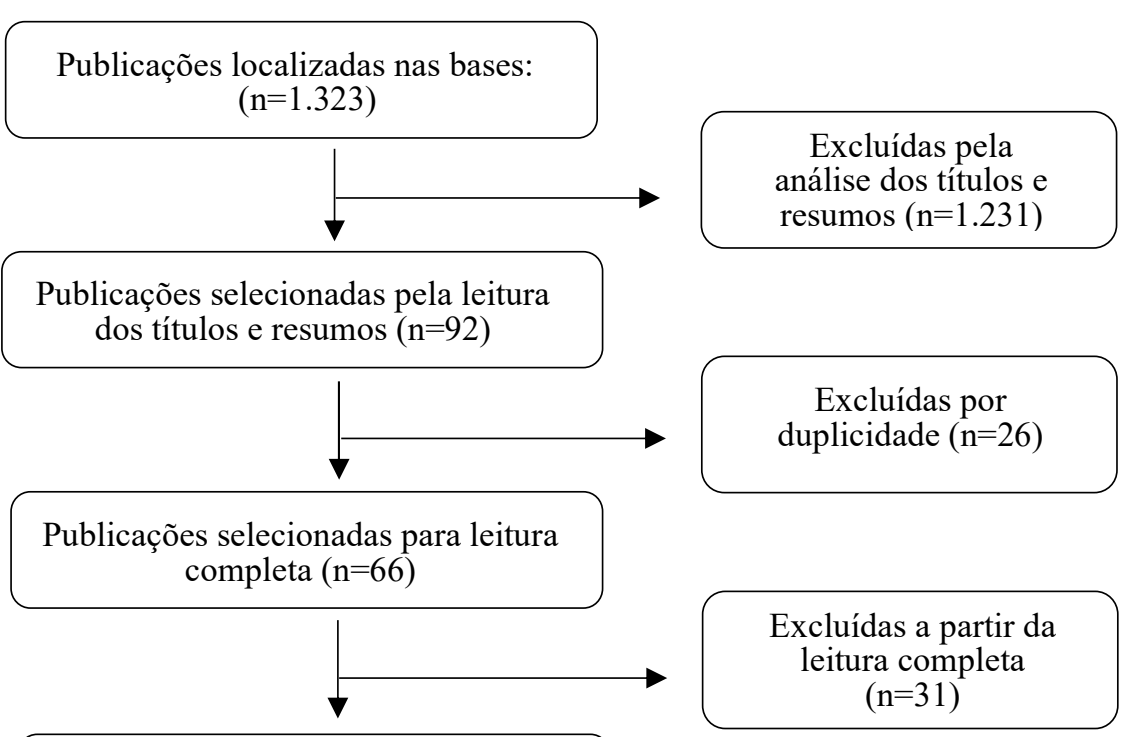

Publicações incluídas $(\mathrm{n}=35)$

Fonte: Autores. 


\section{Etapas 6-9: Mapeamento, coleta, análise e resumo dos dados}

Os artigos foram analisados mediante indicadores de coleta de dados digitados em planilhas eletrônicas disponíveis no programa Microsoft Excel 365®, designados por: ano de publicação, país de origem, título, autores, área de conhecimento, objetivo, população de estudo/amostra, abordagem do estudo, resultados principais e implicações relacionadas ao cuidado interprofissional.

Os resultados foram apresentados por meio de técnicas descritivas: distribuição de estudos por período de publicação, abordagem de estudo, país de origem e área de conhecimento. As características dos estudos foram apresentadas em quadro síntese. Resumiram-se as evidências em relação ao objetivo da revisão, tirando-se conclusões e observando quaisquer implicações dos resultados. Para análise de série temporal das produções, empregou-se a regressão de PraisWinsten (ANTUNES; CARDOSO, 2015).

\section{Resultados}

As 35 publicações incluídas nesta revisão foram divulgadas entre 2009 e 2019. Verifica-se tendência estacionária das publicações sobre o cuidado interprofissional à criança e ao adolescente no período (VPA=3,24; $\left.\mathrm{IC}_{95 \%}=-14,10 ; 24,08\right)$. $\mathrm{O}$ ano em que houve mais publicações de artigos foi em $2017(\mathrm{n}=9 ; 25,71 \%)$. O quantitativo de produção anual e acumulada dos últimos 10 anos pode ser visualizado na Figura 2.

Figura 2 - Produção científica sobre o cuidado interprofissional à criança e ao adolescente de 2009 até setembro de 2019.

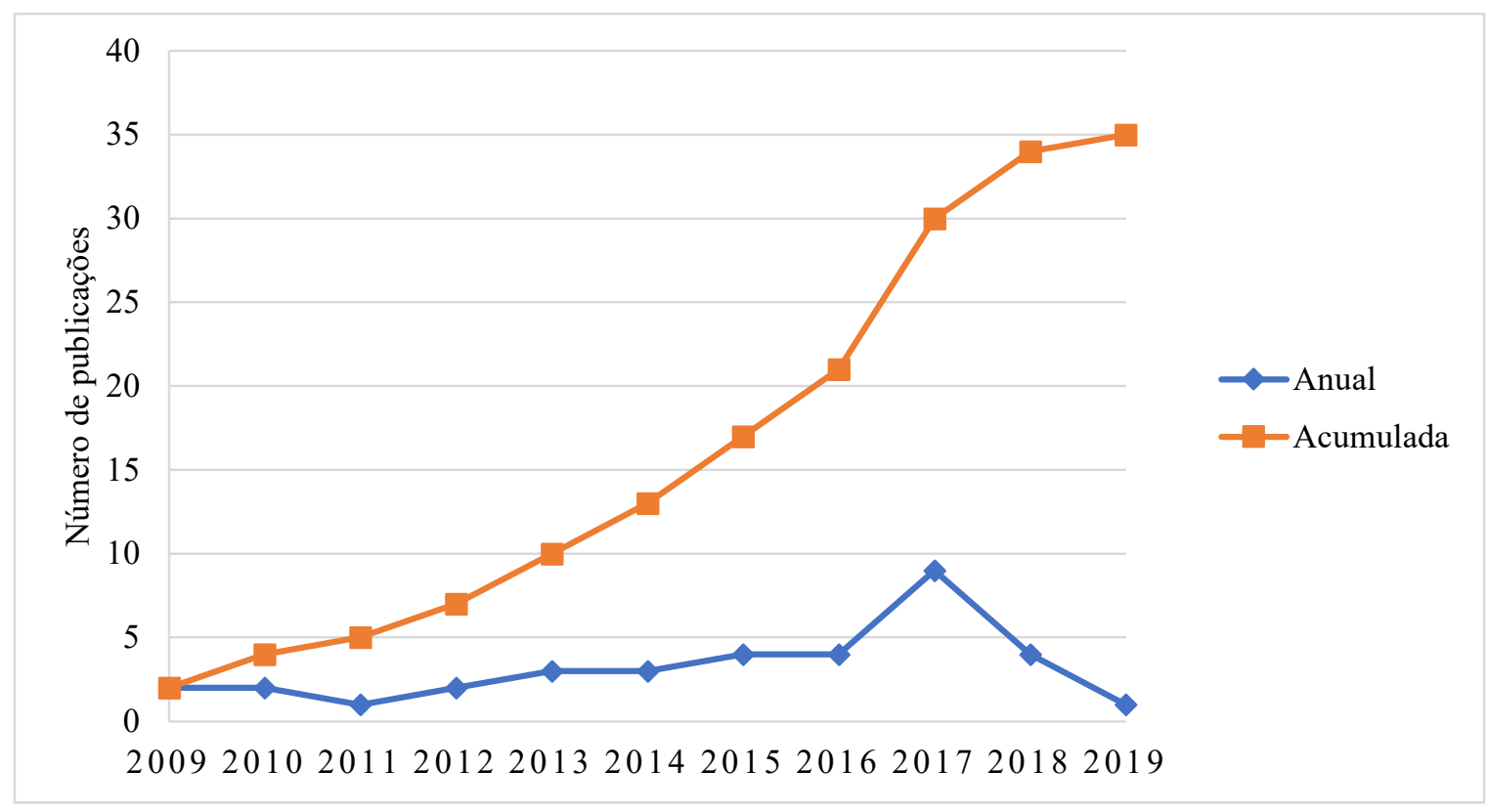

Fonte: Autores 
Destaca-se o predomínio de estudos qualitativos $(n=20 ; 57,14 \%)$, seguido de estudos quantitativos $(\mathrm{n}=9 ; 25,72 \%)$ e estudos de métodos mistos $(\mathrm{n}=6 ; 17,14 \%)$. Com relação aos países responsáveis por tais publicações, constatou-se maior proporção nos Estados Unidos $(n=11 ; 31,43 \%)$ e, consecutivamente, pela Noruega $(n=7 ; 20,00 \%)$, Canadá $(n=5 ; 14,29 \%)$, Austrália $(n=4 ; 11,43 \%)$, Suécia $(n=4 ; 11,43 \%)$, Hong Kong $(n=1 ; 2,86 \%)$, Inglaterra $(n=1$; $2,86 \%)$, Irlanda do Norte ( $n=1 ; 2,86 \%)$ e Japão $(n=1 ; 2,86 \%)$.

No tocante às áreas de atuação dos autores principais dos artigos, verificou-se que a Enfermagem foi a área que mais publicou sobre a temática $(\mathrm{n}=14 ; 40,00 \%)$, superando a Psicologia $(\mathrm{n}=6 ; 17,14 \%)$, o Serviço Social $(\mathrm{n}=4 ; 11,43 \%)$, a Medicina $(\mathrm{n}=3 ; 8,57 \%)$, a Fisioterapia $(\mathrm{n}=2 ; 5,71 \%)$, a Terapia Ocupacional $(\mathrm{n}=2 ; 5,71 \%)$, a Educação $(\mathrm{n}=1 ; 2,86 \%)$, as Ciências Sociais $(n=1 ; 2,86 \%)$, a Farmácia $(n=1 ; 2,86 \%)$ e a Fonoaudiologia $(n=1 ; 2,86 \%)$. Observouse que $54,29 \%(n=19)$ dos artigos foram oriundos de produção colaborativa com outros profissionais e 45,71\% (n=16) de produção uniprofissional. Quadro 1.

As características das publicações incluídas nesta revisão se encontram dispostas no

Quadro 1 - Apresentação das características das publicações incluídas na revisão.

\begin{tabular}{|c|l|c|c|c|}
\hline & \multicolumn{1}{|c|}{ Título } & Autores (ano) & País & $\begin{array}{c}\text { Abordagem } \\
\text { do estudo }\end{array}$ \\
\hline A1 & $\begin{array}{l}\text { A hermeneutic study of integrating psychotherapist } \\
\text { competence in postnatal child health care: nurses' } \\
\text { perspectives }\end{array}$ & $\begin{array}{c}\text { Kornaros } \text { et al. } \\
(2018)\end{array}$ & Suécia & Qualitativo \\
\hline A2 & $\begin{array}{l}\text { An interprofessional exploration of nursing and } \\
\text { social work roles when working jointly with } \\
\text { families }\end{array}$ & $\begin{array}{c}\text { Bennett } \text { et al. } \\
(2016)\end{array}$ & Austrália & Qualitativo \\
\hline A3 & $\begin{array}{l}\text { Caring For Kids Where They Live: Interprofessional } \\
\text { collaboration in teaching and learning in school } \\
\text { settings }\end{array}$ & $\begin{array}{c}\text { Ogenchuk, } \\
\text { Spurr, \& Bally } \\
(2013)\end{array}$ & Canadá & Qualitativo \\
\hline A4 & $\begin{array}{l}\text { Community-Based Learning Collaboratives and } \\
\text { Participant Reports of Interprofessional } \\
\text { Collaboration, Barriers to, and Utilization of } \\
\text { Child Trauma Services }\end{array}$ & $\begin{array}{c}\text { Hanson } \text { et al. } \\
(2018)\end{array}$ & $\begin{array}{l}\text { Estados } \\
\text { Unidos }\end{array}$ & Quantitativo \\
\hline A5 & $\begin{array}{l}\text { Developing a Physical Activity and Nutrition } \\
\text { After-School Program With Youth at Risk }\end{array}$ & $\begin{array}{c}\text { DeVries } \\
(2016)\end{array}$ & $\begin{array}{l}\text { Estados } \\
\text { Unidos }\end{array}$ & Qualitativo \\
\hline
\end{tabular}




\begin{tabular}{|c|c|c|c|c|}
\hline A6 & $\begin{array}{l}\text { Healthy Kids: Impacting Children's Health in } \\
\text { Rural Alabama }\end{array}$ & $\begin{array}{l}\text { Langham et } \\
\text { al. (2017) }\end{array}$ & $\begin{array}{l}\text { Estados } \\
\text { Unidos }\end{array}$ & Qualitativo \\
\hline A7 & $\begin{array}{l}\text { Implications for school nursing through } \\
\text { interprofessional education and practice }\end{array}$ & $\begin{array}{l}\text { Lam, Chan, \& } \\
\text { Yeung (2013) }\end{array}$ & Hong Kong & Qualitativo \\
\hline A8 & $\begin{array}{l}\text { Improving interprofessional collaboration in a } \\
\text { community setting: relationships with burnout, } \\
\text { engagement and service quality }\end{array}$ & $\begin{array}{l}\text { Martinussen et } \\
\text { al. (2012) }\end{array}$ & Noruega & Quantitativo \\
\hline A9 & $\begin{array}{l}\text { Interprofessional collaboration at transition of } \\
\text { care: perspectives of child and family health } \\
\text { nurses and midwives }\end{array}$ & $\begin{array}{l}\text { Psaila et al. } \\
\qquad \text { (2014) }\end{array}$ & Austrália & Misto \\
\hline A 10 & $\begin{array}{l}\text { Interprofessional Collaboration to Support } \\
\text { Children with Diabetes }\end{array}$ & $\begin{array}{l}\text { Polo, \& Cahill } \\
\text { (2017) }\end{array}$ & $\begin{array}{l}\text { Estados } \\
\text { Unidos }\end{array}$ & Quantitativo \\
\hline A11 & $\begin{array}{l}\text { Interprofessional collaboration: self-reported } \\
\text { successful collaboration by teachers and social } \\
\text { workers in multidisciplinary teams }\end{array}$ & $\begin{array}{l}\text { Hesjedal, } \\
\text { Hetland, \& } \\
\text { Iversen }(2015)\end{array}$ & Noruega & Qualitativo \\
\hline A 12 & $\begin{array}{l}\text { Prewarming in a Pediatric Hospital: Process } \\
\text { Improvement Through Interprofessional } \\
\text { Collaboration }\end{array}$ & $\begin{array}{l}\text { Tveit, Belew, } \\
\text { \& Noble } \\
(2015)\end{array}$ & $\begin{array}{l}\text { Estados } \\
\text { Unidos }\end{array}$ & Qualitativo \\
\hline A13 & $\begin{array}{l}\text { Reorganisation of healthcare services for } \\
\text { children and families: Improving collaboration, } \\
\text { service quality, and worker well-being }\end{array}$ & $\begin{array}{l}\text { Martinussenet } \\
\text { al. (2017) }\end{array}$ & Noruega & Quantitativa \\
\hline A14 & $\begin{array}{l}\text { Teaming Up in Child Welfare: The Perspective } \\
\text { of Guardians ad Litem on the Components of } \\
\text { Interprofessional Collaboration }\end{array}$ & $\begin{array}{c}\text { Phillips, \& } \\
\text { Walsh (2019) }\end{array}$ & $\begin{array}{l}\text { Estados } \\
\text { Unidos }\end{array}$ & Qualitativo \\
\hline A 15 & $\begin{array}{l}\text { What do we think about them and what do they } \\
\text { think about us? Social representations of } \\
\text { interprofessional and interorganizational } \\
\text { collaboration in the welfare sector }\end{array}$ & $\begin{array}{l}\text { Widmark et } \\
\text { al. (2016) }\end{array}$ & Suécia & Qualitativo \\
\hline
\end{tabular}




\begin{tabular}{|c|c|c|c|c|}
\hline A16 & $\begin{array}{l}\text { A process oriented approach to promoting } \\
\text { collaborative practice: Incorporating complexity } \\
\text { Methods }\end{array}$ & $\begin{array}{l}\text { Solomon, \& } \\
\text { Risdon (2014) }\end{array}$ & Canadá & Qualitativo \\
\hline A17 & $\begin{array}{l}\text { Public health nurses' perceptions of interprofessional } \\
\text { collaboration related to adolescents' mental } \\
\text { health problems in secondary schools: A } \\
\text { phenomenographic study }\end{array}$ & $\begin{array}{l}\text { Granrud et al. } \\
\qquad(2019)\end{array}$ & Noruega & Qualitativo \\
\hline A18 & $\begin{array}{l}\text { A Service-Learning Project to Eliminate } \\
\text { Barriers to Oral Care for Children With Special } \\
\text { Health Care Needs }\end{array}$ & $\begin{array}{l}\text { Demattei, \& } \\
\text { Goss (2012) }\end{array}$ & $\begin{array}{l}\text { Estados } \\
\text { Unidos }\end{array}$ & Misto \\
\hline A19 & $\begin{array}{l}\text { Collaborating across the threshold: The } \\
\text { development of interprofessional expertise in } \\
\text { child safeguarding }\end{array}$ & $\begin{array}{l}\text { Hood et al. } \\
\quad(2017)\end{array}$ & Inglaterra & Qualitativo \\
\hline A20 & $\begin{array}{l}\text { Collaboration between teachers and speech and } \\
\text { language therapists: Services for primary } \\
\text { school children with speech, language and } \\
\text { communication needs }\end{array}$ & $\begin{array}{l}\text { Glover, } \\
\text { McCormack, } \\
\text { \& Smith- } \\
\text { Tamaray } \\
\text { (2015) }\end{array}$ & Austrália & Misto \\
\hline A 21 & $\begin{array}{l}\text { Implementing a collaborative coaching } \\
\text { intervention for professionals providing care to } \\
\text { children and their families: An exploratory study }\end{array}$ & $\begin{array}{l}\text { Tatla et al. } \\
\text { (2017) }\end{array}$ & Canadá & Misto \\
\hline $\mathrm{A} 22$ & $\begin{array}{l}\text { Interprofessional Collaboration Among Physical } \\
\text { Therapy, Speech-Language Pathology, and } \\
\text { Engineering Faculty and Students to Address } \\
\text { Global Pediatric Rehabilitation Needs: A Case } \\
\text { Report }\end{array}$ & $\begin{array}{l}\text { Hayward et al. } \\
\quad(2016)\end{array}$ & $\begin{array}{l}\text { Estados } \\
\text { Unidos }\end{array}$ & Qualitativo \\
\hline A23 & $\begin{array}{l}\text { Interprofessional education internships in } \\
\text { schools: jump starting change }\end{array}$ & $\begin{array}{l}\text { Salm et al. } \\
\quad(2010)\end{array}$ & Canadá & Qualitativo \\
\hline A24 & $\begin{array}{l}\text { Interprofessional teamwork in Swedish pediatric } \\
\text { cardiology: A national exploratory study }\end{array}$ & $\begin{array}{l}\text { Birkeland et } \\
\text { al. (2013) }\end{array}$ & Suécia & Misto \\
\hline A25 & $\begin{array}{l}\text { Patient safety culture transformation in a } \\
\text { children's hospital: an interprofessional approach }\end{array}$ & $\begin{array}{l}\text { Nagelkerk et } \\
\text { al. }(2014)\end{array}$ & $\begin{array}{l}\text { Estados } \\
\text { Unidos }\end{array}$ & Quantitativo \\
\hline
\end{tabular}




\begin{tabular}{|c|c|c|c|c|}
\hline A26 & $\begin{array}{l}\text { Perceptions of interprofessional collaboration } \\
\text { within child mental health care in Norway }\end{array}$ & $\begin{array}{l}\text { Odegard, \& } \\
\text { Strype (2009) }\end{array}$ & Noruega & Quantitativo \\
\hline A27 & $\begin{array}{l}\text { Professional collaboration -- support for children } \\
\text { with cancer and their families -- focus group } \\
\text { interview -- a source of information and } \\
\text { knowledge -- professionals' perspectives } \\
\text { (EILERTSEN et al., 2009) }\end{array}$ & $\begin{array}{l}\text { Eilertsen et al. } \\
\qquad(2009)\end{array}$ & Noruega & Qualitativo \\
\hline A28 & $\begin{array}{l}\text { Service user participation in interprofessional } \\
\text { teams in child welfare in Norway: vulnerable } \\
\text { adolescents' perceptions }\end{array}$ & $\begin{array}{l}\text { Sæbjørnsen, \& } \\
\text { Willumsen } \\
\text { (2015) }\end{array}$ & Noruega & Qualitativo \\
\hline A29 & $\begin{array}{l}\text { Shared responsibility: school nurses' experience } \\
\text { of collaborating in school-based interprofessional } \\
\text { teams }\end{array}$ & $\begin{array}{l}\text { Reutersward, } \\
\text { \& Hylander } \\
\quad(2017)\end{array}$ & Suécia & Qualitativo \\
\hline A30 & $\begin{array}{l}\text { Descriptive study of interprofessional collaboration } \\
\text { competency scale for children with medical } \\
\text { complexity }\end{array}$ & $\begin{array}{l}\text { Shimmura, \& } \\
\text { Tadaka (2018) }\end{array}$ & Japão & Quantitativo \\
\hline A31 & $\begin{array}{l}\text { Descriptive study of interprofessional collaboration } \\
\text { between physicians and osteopaths for the } \\
\text { pediatric population in Quebec, Canadá }\end{array}$ & $\begin{array}{l}\text { Morin, } \\
\text { Desrosiers, \& } \\
\text { Gaboury } \\
\text { (2017) }\end{array}$ & Canadá & Quantitativo \\
\hline A 32 & $\begin{array}{l}\text { Listening to paediatric primary care nurses: a } \\
\text { qualitative study of the potential for interprofessional } \\
\text { oral health practice in six federally qualified } \\
\text { health centres in Massachusetts and Maryland }\end{array}$ & $\begin{array}{l}\text { Bernstein et } \\
\text { al. }(2017)\end{array}$ & $\begin{array}{l}\text { Estados } \\
\text { Unidos }\end{array}$ & Qualitativo \\
\hline A33 & $\begin{array}{l}\text { Is it time to talk? Understanding specialty child } \\
\text { mental healthcare providers' decisions to engage in } \\
\text { interdisciplinary communication with pediatricians }\end{array}$ & $\begin{array}{l}\text { Reiss, Greene, } \\
\& \text { Ford (2017) }\end{array}$ & $\begin{array}{l}\text { Estados } \\
\text { Unidos }\end{array}$ & Qualitativo \\
\hline A34 & $\begin{array}{l}\text { An interprofessional learning module on asthma } \\
\text { health promotion }\end{array}$ & $\begin{array}{l}\text { Saini et al. } \\
\quad(2011)\end{array}$ & Austrália & Quantitativo \\
\hline A35 & $\begin{array}{l}\text { An interprofessional approach to improving } \\
\text { paediatric medication safety }\end{array}$ & $\begin{array}{l}\text { Stewart et al. } \\
\qquad(2010)\end{array}$ & $\begin{array}{l}\text { Irlanda do } \\
\text { Norte }\end{array}$ & Misto \\
\hline
\end{tabular}

Fonte: Autores 
De acordo com os conteúdos dos artigos incluídos na revisão, foi possível estabelecer três categorias para apresentação do mapeamento descritivo dos dados, a saber: a) A importância da EIP para o cuidado colaborativo em pediatria; b) Potencialidades e desafios da CIP em pediatria; e, c) As implicações do cuidado interprofissional à criança e ao adolescente.

\section{A importância da EIP para o cuidado colaborativo em pediatria}

Os estudos demonstraram o potencial da EIP em pediatria, devido à oportunidade de aprendizagem colaborativa que ela oferece aos envolvidos, com o desenvolvimento de competências interprofissionais para o atendimento integral de crianças e adolescentes. Os principais aspectos aprimorados durante as experiências de aprendizagem colaborativa elencados pelas pesquisas foram: a comunicação interprofissional, o reconhecimento da importância das outras profissões, a clareza dos papéis profissionais, o estabelecimento de objetivos compartilhados, a construção de senso de trabalho em equipe e a atenção centrada na pessoa (A4; A5; A6; A22; A23; A25; A32; A34; A35).

São várias as experiências de aprendizagem interprofissional ilustradas nas publicações identificadas, como em módulos de aprendizagem nos cursos da área de saúde, estágios, programas de base comunitária e treinamento/capacitação da equipe de saúde.

Com a intenção de propulsionar a EIP, alguns autores (A22; A34; A35) desenvolveram módulos de aprendizagem interprofissional em cursos de graduação da área da saúde, os quais permitiram aos estudantes vivenciarem essa relação teórico-prática, em um processo de instrução colaborativa e desenvolvimento de competências essenciais como comunicação e respeito mútuo.

No A34, o módulo de aprendizagem interprofissional focado na promoção à saúde em asma, por exemplo, foi realizado em três dias com estudantes dos cursos de medicina, enfermagem e farmácia da Universidade de Sydney, na Austrália. Após essa experiência, verificou-se que houve mudança significativa no subtópico "Trabalho em Equipe" da Escala Prontidão para Aprendizagem Interprofissional ao comparar os momentos pré e pós módulo. Outro estudo (A35), ao realizar um workshop interprofissional de ensino-aprendizagem sobre prescrição e administração de medicamentos em pediatria com estudantes de medicina e enfermagem, verificou que houve melhora no conhecimento sobre a segurança dos pacientes pediátricos no tocante à medicação. Também identificou-se que a aprendizagem ocorreu de forma mais eficaz devido à participação de vários profissionais.

Estágios interprofissionais no ambiente escolar foram implementados com a finalidade de proporcionar aos estudantes a experiência de colaboração interprofissional (A3; A23). O estágio clínico pediátrico interprofissional descrito no A3 se mostrou como uma maneira eficaz de aprender com, de e sobre as outras profissões. Ele possibilitou o desenvolvimento do respeito mútuo, da resolução de problemas em conjunto e uma visão holística das crianças e dos adolescentes, considerando a unidade familiar. Pesquisadores do A7 enfatizam a importância do envolvimento de mais de uma profissão no atendimento às necessidades das crianças e adolescentes, pois apenas um profissional não é suficiente para compreender e atender toda a complexidade de saúde existente nesse ciclo da vida. 
Além disso, existem outras experiências exitosas evidenciadas nos artigos incluídos nessa revisão, também desenvolvidas com estudantes. Destacam-se os programas interprofissionais de base comunitária e escolar, como o "Kick and Cook-a-Palooza" (A5) e "Crianças Saudáveis" (A6). Este foi desenvolvido por uma equipe interprofissional com a finalidade de auxiliar enfermeiras escolares na triagem de saúde de crianças e adolescentes, enquanto o programa "Kick and Cook-a-Palooza" visou ensinar hábitos saudáveis e a importância de um estilo de vida positivo às crianças e adolescentes em situação de risco, através de atividades recreativa após o horário escolar. Essas experiências são de grande importância, uma vez que proporcionaram aos estudantes um ambiente de atuação interprofissional e um senso de trabalho em equipe que os acompanhará após a graduação, em suas carreiras profissionais.

Uma das estratégias utilizadas para fomentar a aprendizagem colaborativa nos serviços de saúde foi a "Aprendizagem Colaborativa baseada na Comunidade" (CBLC) (A4). Esta foi desenvolvida com a finalidade de avaliar a CIP, as barreiras e utilização dos serviços de trauma infantil antes e após uma intervenção educativa. Foram aplicados questionários pré e pós sessão de aprendizagem, em que, após a CBLC a CIP aumentou em 12\% de acordo com os seguintes itens: trabalho em conjunto para que os clientes não caiam em lacunas do sistema, compartilhamento de informações de avaliação e tratamento, e garantia de conclusão do tratamento dos clientes. Assim, os pesquisadores apontaram que as barreiras no tratamento do trauma infantil diminuíram em 16\% após a intervenção e houve um aumento na utilização do serviço em 5\%.

Ainda, outra maneira de desenvolver a EIP foi através de treinamentos aos profissionais de saúde e estudantes (A8; A21; A25) visando o aprimoramento de saberes da prática interprofissional. No A8, por exemplo, os autores realizaram uma intervenção com o propósito de melhorar a colaboração entre os membros de equipes de saúde, em que a população de estudo foi dividida em dois grupos: o de intervenção e outro de comparação, ao passo que, no grupo intervenção, os participantes receberam cursos para trabalhar com crianças à luz da interprofissionalidade e intersetorialidade, como cursos de colaboração entre serviços, treinamento no uso de instrumentos de medição e intervenções específicas, como lidar com filhos de pais com doença mental e prevenção do uso de álcool entre adolescentes. A partir dessa experiência, foi verificado que a colaboração entre os profissionais do distrito modelo aumentou no grupo intervenção em relação ao de comparação, que não recebeu a intervenção.

Neste contexto, é importante conhecer as percepções de profissionais de saúde, estudantes e usuários do serviço sobre as potencialidades e desafios do cuidado colaborativo em pediatria, a fim de aperfeiçoar as estratégias da EIP existentes e repensar outras formas de fomentar a aprendizagem interprofissional.

\section{Potencialidades e desafios da CIP em pediatria}

Em estudos analisados, os profissionais de saúde destacaram a importância da CIP para melhorar o atendimento às crianças e famílias (A1; A9; A10; A17). Enfermeiras e parteiras de serviços de saúde materno-infantil, participantes do estudo A9, relataram o impacto negativo da desconexão dos serviços e pontuaram a CIP como conceito-chave para garantir $\mathrm{o}$ atendimento em rede entre a maternidade e as unidades de saúde da família, favorecendo a 
continuidade dos cuidados. Dessa forma, as parteiras e enfermeiras afirmaram que as práticas de saúde pautadas no cuidado colaborativo facilitam as relações com as famílias e aumentam as chances de manutenção de vínculos entres os intervenientes do cuidado.

Resultado semelhante foi identificado no estudo A10, que investigou a percepção de enfermeiros atuantes em escolas sobre a CIP para apoiar a promoção da saúde e o desenvolvimento de habilidades de autogestão de escolares com diabetes. Entre os enfermeiros, 91,8\% citaram a CIP como ferramenta valiosa para auxiliar nessa autogestão realizada de forma colaborativa.

Nota-se a relevância da comunicação na CIP para um bom desenvolvimento da equipe, bem como a necessidade de uma "linguagem comum" para facilitar o diálogo entre os profissionais e o compartilhamento de informações no cuidado em pediatria (A2; A14; A19). De acordo com estudantes de fisioterapia e engenharia, para o trabalho interprofissional ser efetivo, os profissionais envolvidos devem estar abertos a ouvir outras opiniões e terem vontade de colaborar com outras pessoas para atingirem um objetivo comum centrado no cliente e família (A22).

Neste sentido, a pesquisa A33 verificou que as crenças positivas dos profissionais sobre o cuidado pediátrico colaborativo é um fator primordial para que se inicie a comunicação interprofissional. As reuniões regulares da equipe (A2) e o feedback dos profissionais sobre a evolução do paciente (A1) também foram citados pelos profissionais como pontos relevantes para a efetivação da prática colaborativa.

O modelo teórico "Percepção de Modelo de Colaboração Interprofissional (PINCOM)" foi utilizado no A26 para analisar as percepções da colaboração interprofissional em três aspectos individual, de grupo e organizacional - possibilitando evidenciar que os elementos mais proeminentes na visão dos profissionais foram: a motivação, a liderança e a cultura organizacional. Para eles, a aplicação desse modelo teórico foi eficaz na melhoria da CIP na prática clínica, pois indica formas de aprimorá-la considerando as percepções dos profissionais da equipe.

Nesta perspectiva, outro estudo (A27) analisou as percepções de profissionais sobre o uso do modelo colaborativo profissional $(\mathrm{MCP})$ para o acompanhamento satisfatório às crianças com câncer e suas famílias. Os autores identificaram que, para os profissionais, o MCP configurouse como um modelo valioso para oferecer suporte a essa população, pois ofereceu segurança aos pais, uma vez que perceberam que toda equipe estava trabalhando em prol de um objetivo comum. Ademais, eles afirmaram que o trabalho interprofissional contribuiu para a ampliação do olhar sobre determinados assuntos e compartilhamento dos saberes interdisciplinares. A esse respeito, ressaltam que a reunião colaborativa se mostrou uma estratégia facilitadora para o trabalho em equipe, haja vista que há troca de informações e corresponsabilização de todos os profissionais sobre os casos clínicos.

Os estudos A11 e A14 apresentam outros facilitadores para o desenvolvimento da CIP em prol do bem-estar infantojuvenil, como o compromisso pessoal, a criação de uma atmosfera positiva, a resolução de problemas de forma colaborativa, o respeito mútuo, o esclarecimento de papéis, o estabelecimento de metas compartilhadas e a confiança mútua. Portanto, a CIP foi apontada como um processo complexo, pois abrange diversos componentes, correlacionados entres si, para que ocorra de maneira profícua. De acordo com os pesquisadores, tais aspectos 
facilitadores permitem que os profissionais de saúde atuem de forma centrada no cliente pediátrico e família.

Em contrapartida, outros sinalizam alguns obstáculos para a CIP, as quais acarretam, principalmente, a fragmentação da assistência à saúde e a acentuação da prática uniprofissional. (A9; A12; A15; A16; A24; A32). Eles apontam como entraves: dificuldade de comunicação, barreiras organizacionais, falta de apoio político e administrativo, colaboração problemática e complexa, falta de estrutura, sinergia da equipe comprometida, falta de gerenciamento de funções e horários divergentes entre os profissionais. Assim, essas barreiras precisam ser superadas para que haja o desenvolvimento da CIP, tendo em vista as suas potencialidades para melhoria na qualidade da atenção integral ao cliente pediátrico e família.

Segundo profissionais, o contato com outros membros da equipe deveria ser contínuo e regular, de modo que houvesse uma comunicação eficaz entre eles, para assim melhorar a qualidade na assistência (A20). Ao verificar a percepção de enfermeiros escolares integrantes de uma equipe interprofissional, pesquisadores do A29 perceberam que eles não tinham clareza em relação ao seu papel dentro da equipe, o que dificultou o trabalho. Esse esclarecimento é imprescindível para a obtenção de bons resultados a partir do trabalho colaborativo.

Em outra pesquisa (A28), foi possível investigar a percepção dos adolescentes sobre a participação em equipes interprofissionais. Os pesquisadores identificaram que, na opinião dos adolescentes, para uma participação exitosa nessas equipes, algumas mudanças precisariam acontecer, como o estabelecimento de uma relação de confiança entre os profissionais e os adolescentes, com a oferta de todas as informações necessárias para uma participação mais ativa e a valorização de suas opiniões. Assim, os autores destacam a importância da inserção dos clientes nas equipes para melhorar os resultados no bem-estar através do suporte interprofissional.

\section{As implicações do cuidado interprofissional à criança e ao adolescente}

Na busca por melhorias na atenção à saúde de crianças e adolescentes, o trabalho interprofissional apresenta potencial para ampliar o cuidado em saúde, considerando a sua complexidade, a justiça social e a busca de construção de novas visões do mundo, conforme pesquisadores do A3.

Em alguns estudos analisados (A3; A6; A7), a escola foi assinalada como ambiente ideal para promoção, avaliação e prevenção à saúde desse público, à luz da práxis interprofissional. O modelo de trabalho na escola parte da compreensão das necessidades de crianças e adolescentes pelos profissionais de saúde, e busca a tomada de decisão conjunta com a escola e escolares para melhorias das condições de saúde dessa população. É possível superar doenças agudas ou conviver com uma doença crônica com maior qualidade de vida a partir do cuidado colaborativo (A3; A13). Além disso, este cuidado promove uma abordagem mais ampla das disparidades na saúde dos escolares, identificando precocemente as situações-problema e estabelecendo intervenções eficazes de acordo com evidências científicas (A6; A7).

A CIP implica no modo de cuidar em saúde, por fundamentar-se na interação dos profissionais com o usuário e a família na busca de um plano de cuidados coletivo. Por isso, alguns estudos propuseram a realização de reuniões da equipe com todos os intervenientes (A27; 
A28; A30). Tal atitude promove uma participação ativa da criança/adolescente e família, o estabelecimento da confiança na equipe e do vínculo, fundamentais para realização de todos os processos necessários no sistema de cuidado em saúde (A27).

O uso de uma escala também contribuiu para melhorar a eficácia da colaboração interprofissional $\mathrm{e}$, consequentemente, $\mathrm{o}$ atendimento às crianças e suas família. Isso ocorre devido à avaliação mais ampliada dos processos saúde-doença e do estabelecimento de uma rede criativa de interação entre todos, propulsora de um cuidado bem-sucedido (A30). Ademais, contribuiu para o reconhecimento da complementaridade de todos os envolvidos no ato de cuidar (A31).

Pesquisadores desenvolveram o estudo A18 com o intuito de promover o bem-estar oral de crianças com alteração de funcionalidade através do cuidado colaborativo entre enfermeiros escolares, professores da escola, um dentista e estudantes de um programa universitário de higiene bucal. Como resultado do estudo, identificou-se que $63,7 \%$ das crianças tiveram comportamento aceitável e cumpriram as instruções adequadas após a intervenção colaborativa. Logo, os pesquisadores apontaram que a CIP resultou em menores índices de doença bucal, pois se estabeleceu maior confiança entre profissionais e usuários.

A interprofissionalidade em saúde extrapola a relação entre profissionais de saúde, contemplando outras áreas do conhecimento que propulsionam melhorias nos cuidados de saúde. Tanto que a pesquisa A22 realizada com fisioterapeutas, fonoaudiólogo e engenheiros descreveu a produção de dois dispositivos de comunicação para crianças que possuíam apraxia Os pesquisadores destacaram que foram elencados, pelos envolvidos, vários benefícios da colaboração, entre eles: importância de ouvir sugestões, desenvolvimento de habilidades para resolução de problemas, comunicação efetiva entre os profissionais e importância da atenção centrada no cliente pediátrico e família.

\section{Discussão}

Com esta revisão, identificou-se que as evidências sobre o cuidado interprofissional às crianças e aos adolescentes são prevalentemente oriundas de estudos qualitativos, desenvolvidos por profissionais da área de enfermagem e de forma colaborativa com outros profissionais. Diversos países pesquisaram sobre a temática nos últimos dez anos, no entanto, não foram encontrados registros nos países da América do Sul. O conteúdo dos artigos incluídos neste estudo permitiu uma melhor compreensão sobre a importância da EIP para a CIP em pediatria, as potencialidades e desafios da CIP em pediatria e as implicações desse cuidado interporfissional à criança e ao adolescente.

Quanto ao predomínio de estudos qualitativos nesta revisão, destaca-se o fato de que a maioria dos artigos abordou aspectos subjetivos do processo de trabalho interprofissional, como as percepções dos profissionais, usuários e família sobre o desenvolvimento da CIP, assim como as competências interprofissionais desenvolvidas e iniciativas de EIP. Portanto, o método qualitativo possibilita a melhor compreensão das experiências humanas, do desenvolvimento de intervenções, das barreiras e facilitadores da implementação dessas intervenções, além de fornecer insights (DENNY; WECKESSER, 2018). 
O predomínio de estudos desenvolvidos nos Estados Unidos advém do grande número de iniciativas sobre a EIP no país. De acordo com estudo anterior, os Estados Unidos, juntamente com outros países desenvolvidos como Canadá e países europeus, possuem mais incentivos para a EIP (HERATH et al., 2017). Por mais que nos últimos anos tenham ocorrido avanços na incorporação da EIP nas políticas de recursos humanos para a saúde nos países da região das Américas, não foram identificados estudos que respondessem à questão de pesquisa pela presente revisão.

Outro ponto a ser discutido relaciona-se à área de atuação dos autores principais dos artigos, ao passo que a enfermagem se revelou como a área com mais publicações acerca da temática. Para Souza e colaboradores (2016), a enfermagem se destaca entre as profissões pelo grande potencial em articular o cuidado de saúde e talvez por isso esteja tão envolvida em pesquisas sobre a temática. Em alguns cenários, é a profissão que se mantém, durante as 24 horas do dia, ao lado do paciente, em uma assistência contínua, que requer uma abordagem holística a partir da CIP para alçar o sucesso prático.

Embora os artigos analisados no presente estudo tenham abordado o tema da interporfissionalidade, identificou-se que boa parte foi oriunda de produção uniprofissional. Diante disso, percebem-se os desafios da CIP também no contexto da produção científica, tendo em vista que os profissionais estão habituados a trabalhar nos moldes da uniprofissionalidade (PEDUZZI et al., 2013).

A partir dos resultados desta revisão, vislumbra-se que a EIP é fundamental para capacitar e qualificar os profissionais de saúde para o cuidado colaborativo e integral à saúde da criança e do adolescente. Assim, destaca-se a importância de se impulsionar o desenvolvimento da EIP nos núcleos de formação profissional e serviços de saúde, pois ela visa promover o aprendizado e o trabalho colaborativo entre os estudantes dos diferentes cursos de graduação em saúde e profissionais inseridos nos serviços (PEDUZZI, 2016).

Nesta perspectiva, se reconhece, na proposta de EIP, a relação recíproca de influência entre educação e atenção à saúde, sistema educacional e sistema de saúde, o que é essencial para sistemas de saúde pública, como o Sistema Único de Saúde (SUS) brasileiro, construído e consolidado como espaço de atenção à saúde, educação profissional, gestão e controle social, orientado pelos princípios de integralidade, equidade, universalidade e participação (PEDUZZI, 2016).

Assim como os artigos A3, A6, A22, A23 e A32, outros pesquisadores, ao relatarem a experiência de estudantes que prestaram o cuidado interprofissional a clientes com Doença de Parkinson, também revelaram que este modelo foi aprovado pelos clientes e promoveu um atendimento holístico centrado no cliente (FRIARY et al., 2018). O desenvolvimento interprofissional é uma iniciativa para romper fronteiras disciplinares e melhorar a qualidade do cuidado através da confiança compartilhada e responsabilidade mútua na resolução de problemas (WITT SHERMAN et al., 2017).

À vista disso, evidencia-se a relevância de se investir na EIP para que haja mudanças nas práticas de saúde. As experiências interprofissionais retratadas nos artigos incluídos nesta revisão demonstram a potencialidade da EIP no desenvolvimento de competências para o trabalho interprofissional. Estudo apontou que este modelo de ensino promove mudanças nas 
atitudes dos estudantes após a interação em relação à CIP e funções profissionais, uma vez que relataram maior compreensão sobre os papéis das outras profissões e os benefícios de se trabalhar em equipes interprofissionais (GOULD; DAY; BARTON, 2017), tal como na melhora do trabalho em equipe, funções e responsabilidades profissionais (MUZYK et al., 2017).

Em diversos países, como o Brasil, o desenvolvimento da EIP ainda é tímido, porém significativo. Ela caminha lado a lado aos ideários do sistema público de saúde, o que fortalece e complementa questões como a compreensão da integralidade do cuidado, às necessidades sociais e em saúde da população, além da promoção a ampliação da concepção de saúde (COSTA, 2016). No entanto, apesar dos avanços alcançados em muitos países, ainda há muito a se fazer, de modo a superar as incoerências do modelo hegemônico de formação e prática uniprofissional. É necessário um efetivo diálogo entre os envolvidos na atenção à saúde e formação profissional, a saber: instituição de ensino, estudantes, profissionais, sistema político em todos os níveis de governo, órgãos de regulação das profissionais, usuários e população (CÂMARA et al., 2016).

Políticas indutoras propulsionam a EIP, como o lançamento do Programa de Educação pelo Trabalho para Saúde (PET Saúde/Interprofissionalidade) no Brasil em 2019, o qual teve o objetivo de promover a integração ensino-serviço-comunidade com foco no SUS e implementar a EIP nos Projetos Pedagógicos Curriculares (PPC) nos cursos da área da saúde. Anseia-se que esta edição do programa possa tornar a prática colaborativa uma experiência que perpassa as interfaces entre a educação na saúde, o processo de trabalho e a prática profissional, não se tornando apenas uma vivência pontual, mas se concretizando e se perpetuando na busca constante pela qualificação do cuidado em saúde nas universidades (ALMEIDA; SILVA, 2019).

Neste caminho, é preciso considerar as percepções dos profissionais de saúde e estudantes sobre a CIP. Esta revisão identificou que grande parte dos estudos incluídos (A1, A2, A9, A10, A14, A17 e A19) reforçam que este método de trabalho melhora o atendimento ao cliente pediátrico e família, pois possibilita o maior vínculo com os usuários e uma comunicação facilitada entre os intervenientes do cuidado. Estudo realizado com diversos profissionais da área da saúde do Canadá possibilitou a identificação de percepções sobre características importantes para o apoio da CIP nas equipes de atenção primária, em que se destacaram a comunicação, o cotratamento e as reuniões juntamente com o paciente (DONNELLY et al., 2019).

A falta de CIP, de confiança e de autonomia profissional implicam negativamente na qualidade da parceria estabelecida no trabalho em equipe (GALLAGHER; GALLAGHER, 2012). Para o sucesso prático na área de pediatria, é importante que os profissionais estejam abertos para aceitar visões e papéis diferentes, que reconheçam seus próprios limites e respeitem todas as áreas de conhecimento, proporcionando uma cooperação mútua para desenvolvimento dos cuidados de saúde (KIM et al., 2019)

Tal como citado no A3, A22, A23 e A35, Mahler e colaboradores (2018) também apontam que os estudantes envolvidos em programas de EIP reconhecem a importância da cooperação, comunicação, no qual o trabalho prático gera mudanças positivas relativas à atenção integral dos usuários. 
A partir das percepções dos profissionais da CIP apontadas nesta revisão, entende-se que ainda há grandes desafios para sua implantação nos sistemas de saúde, os quais devem ser superados para que haja melhoria nos cuidados pediátricos. Pesquisa observou que estudantes participantes de uma abordagem para desenvolvimento de uma formação interprofissional na Alemanha descreveram mais benefícios do que dificuldades em relação à aprendizagem conjunta (MAHLER et al., 2018). Os pesquisadores destacaram que foram apontados como desafios: dificuldade de interação entre estudantes de outras profissões da saúde, abordagem dos conteúdos predominantemente de uma profissão de saúde e adversidade em desenvolver uma linguagem comum. Tais obstáculos também foram identificados no A9, A12, A15, A16, A24 e A32, conforme mapeado nos estudos.

Nota-se que, muitas vezes, a matriz curricular dos cursos da área da saúde não proporciona experiências de aprendizado entre estudantes de outros departamentos, limitando este contato apenas às atividades extracurriculares (KIM, 2019). Logo, é necessário a incorporação da EIP nos currículos para o desenvolvimento de competências interprofissionais ainda na academia.

A CIP em pediatria busca potencializar os cuidados de saúde através da compreensão das necessidades infantojuvenil, em que a tomada de decisão é realizada a partir da comunicação efetiva entre os profissionais, cliente pediátrico e família. Este modelo de atenção centrado nesta tríade proporciona um maior desempenho da equipe de saúde e maior qualidade do cuidado (GONZALO et al., 2016). Por isso, precisa ser considerado pelos sistemas de saúde e educação em todo o mundo.

\section{Limitações}

Ressalta-se que, por considerar apenas artigos originais, perde-se o corpo de conhecimento presente na literatura cinzenta, como em teses e trabalhos acadêmicos, informes técnicos ou institucionais não revisados por pares e editorados. Ainda, há outras limitações inerentes ao método, em que relatórios de pesquisas podem não ter sido identificados pela estratégia de busca adotada. $\mathrm{O}$ equilíbrio entre a amplitude dos conteúdos e a profundidade na análise foi um desafio, devido ao grande volume de publicações identificadas.

\section{Conclusão}

Este estudo forneceu o mapeamento das evidências acerca do cuidado interprofissional às crianças e aos adolescentes no contexto global. Acredita-se que este poderá subsidiar novas iniciativas de mudanças na educação e na prática de saúde, por meio da EIP e do CIP, respectivamente. Dentre os resultados apresentados, destaca-se a importância da EIP como propulsora da CIP nos serviços de saúde à criança e ao adolescente, sendo que as principais medidas desenvolvidas para este fim foram os estágios, os módulos de aprendizagem, os treinamentos e os programas de base comunitária.

Além disso, cabe ressaltar a importância de se valorizarem as percepções dos profissionais e estudantes de saúde em equipes interprofissionais no cuidado pediátrico, uma vez 
que a compreensão dos desafios e das potencialidades da CIP permite o aperfeiçoamento do desempenho destas equipes e o maior sucesso prático em pediatria.

Constata-se a necessidade de investimentos no cuidado interprofissional à criança e ao adolescente em países da América do Sul, o que pode ser efetivado a partir de mudanças na educação e na prática, como na produção de um corpo científico mais robusto nesta área de conhecimento. Novas pesquisas precisam ser desenvolvidas, com o intuito de identificar a realidade desses países acerca deste tipo de cuidado e propor novas formas de (ensinar a) cuidar, apoiado em intervenções colaborativas em saúde, além de investigar os seus efeitos na saúde infantojuvenil.

\section{Agradecimento}

Os autores agradecem à Pró-Reitoria de Pesquisa e Pró-Reitoria de Ensino de PósGraduação da Universidade Federal de Mato Grosso pelo apoio à publicação e ao Ministério da Saúde pela contemplação de bolsas pelo "Programa Educação pelo Trabalho para a Saúde - Interprofissionalidade" (PET-Saúde/Interprofissionalidade) da UFMT, via edital 10/2018.

\section{Referências}

ALMEIDA, R. G. S.; SILVA, C. B. G. Interprofessional Education and the advances of Brazil. Revista Latino-Americana de Enfermagem, v. 27, e3152, 2019. DOI: 10.1590/1518-8345.3148-3152. Acesso em: 12 maio 2021.

ANTUNES, J. L. F.; CARDOSO, M. R. A. Using time series analysis in epidemiological studies. Epidemiologia e Serviços de Saúde, v. 24, n. 3, p. 565-576, 2015. DOI: 10.5123/S1679-49742015000300024. Acesso em: 12 maio 2021.

AYRES, J. R. C. M. Care: Work, Interaction and Knowing Health Practices. Rev baiana enferm, v. 31, p. 1-4, 2017. DOI: 10.18471/rbe.v31i1.21847. Acesso em: 12 maio 2021.

BENNETT, E. et al. An interprofessional exploration of nursing and social work roles when working jointly with families. Journal of Interprofessional Care, v. 30, n. 2, p. 232-237, 2016. DOI: 10.3109/13561820.2015.1115755. Acesso em: 12 maio 2021.

BERNSTEIN, J. et al. Listening to paediatric primary care nurses: A qualitative study of the potential for interprofessional oral health practice in six federally qualified health centres in Massachusetts and Maryland. BMJ Open, v. 7, n. 3, e014124, 2017. DOI:

10.1136/bmjopen-2016-014124. Acesso em: 12 maio 2021.

BIRKELAND, A. L. et al. Interprofessional teamwork in Swedish pediatric cardiology: A national exploratory study. Journal of Interprofessional Care, v. 27, n. 4, p. 320-325, 2013. DOI: 10.3109/13561820.2013.767224. Acesso em: 12 maio 2021. 
CÂMARA, A. M. C. S. et al. Interprofessional education in Brazil: building synergic networks of educational and healthcare processes. Interface - Comunicação, Saúde, Educação, v. 20, n. 56, p. 5-8, 2016. Acesso em: 12 maio 2021.

CASANOVA, I. A.; BATISTA, N. A.; MORENO, L. R. Interprofessional education and shared practice in multiprofessional health residency programs. Interface - Comunicação, Saúde, Educação, v. 22, p. 1325-1337, 2018. DOI: 10.1590/1807-57622017.0186. Acesso em: 12 maio 2021.

COSTA, M. V. The interprofessional education in Brazilian context: some reflections.

Interface - Comunicação, Saúde, Educação, v. 20, n. 56, p. 197-198, 2016. Acesso em: 12 maio 2021.

DENNY, E.; WECKESSER, A. Qualitative Research: What It Is and What It Is Not: Study Design: Qualitative Research. BJOG: An International Journal of Obstetrics and Gynaecology, v. 126, n. 3, p. 369, 2018. DOI: 10.1111/1471-0528.15198. Acesso em: 12 maio 2021.

DEMATTEI, R. R.; ALLEN, J.; GOSS, B.A Service-Learning Project to Eliminate Barriers to Oral Care for Children With Special Health Care Needs. Journal of School Nursing, v. 28, n. 3, p. 168-174, 2012. DOI: 10.1177/1059840511432473. Acesso em: 12 maio 2021.

DEVRIES, D. Developing a Physical Activity and Nutrition After-School Program With Youth at Risk. Therapeutic Recreation Journal, v. 50, n. 4, p. 304-326, 2016. DOI: 10.18666/TRJ-2016-V50-I4-7602. Acesso em: 12 maio 2021.

DONNELLY, C. et al. Measuring the performance of interprofessional primary health care teams: understanding the teams perspective. Primary Health Care Research \& Development, v. 20, e125, 2019. DOI: 10.1017/S1463423619000409. Acesso em: 12 maio 2021.

DOMAJNKO, B. et al. Interprofessional education in Europe: policy and practice.

Beyond interprofessionalism: caring together with rather than for people.

Antwerpen/Apeldoorn: Garant; 2015. Acesso em: 12 maio 2021.

EILERTSEN, M. E. B. et al. Professional collaboration - Support for children with cancer and their families - Focus group interview - A source of information and knowledge Professionals' perspectives. Journal of Interprofessional Care, v. 23, n. 4, p. 355-368, 2009. DOI: 10.1080/13561820902881726. Acesso em: 12 maio 2021.

FARIAS, D. N. et al. Interdisciplinaridade E Interprofissionalidade na Estratégia Saúde Da Família. Trabalho, Educação e Saúde, v. 16, n. 1, p. 141-162, 2017. DOI: 10.1590/19817746-sol00098. Acesso em: 12 maio 2021.

FRIARY, P. et al. Navigating Interprofessional Spaces: Experiences of Clients Living with Parkinson's Disease, Students and Clinical Educators. Journal of Interprofessional Care, v. 32, n. 3, p. 304-312, 2018. DOI: 10.1080/13561820.2017.1417238. Acesso em: 12 maio 2021. 
GALLAGHER, R. M.; GALLAGHER, H. C. Improving the working relationship between doctors and pharmacists: Is inter-professional education the answer? Advances in Health Sciences Education: Theory and Practice, v. 17, p. 247-257, 2012. DOI: 10.1007/s10459010-9260-5. Acesso em: 12 maio 2021.

\section{GLOBAL FORUM ON INNOVATION IN HEALTH PROFESSIONAL EDUCATION, BOARD ON GLOBAL HEALTH \& INSTITUTE OF MEDICINE. Interprofessional} Education for Collaboration: Learning How to Improve Health from Interprofessional Models Across the Continuum of Education to Practice: Workshop Summary. Washington (DC): National Academies Press, 2013. DOI: 10.17226/13486. Acesso em: 12 maio 2021.

GLOVER, A.; MCCORMACK, J.; SMITH-TAMARAY, M. Collaboration between teachers and speech and language therapists: Services for primary school children with speech, language and communication needs. Child Language Teaching and Therapy, v. 31, n. 3, p. 363-385, 2015. DOI: 10.1177/0265659015603779. Acesso em: 12 maio 2021.

GONZALO, J. D. et al. Interprofessional collaborative care characteristics and the occurrence of bedside interprofessional rounds: a cross-sectional analysis. BMC Health Services Research, v. 16, n. 1, p. 459, 2016. DOI: 10.1186/s12913-016-1714-x. Acesso em: 12 maio 2021.

GOULD, K.; DAY, K. H.; BARTON, A. T. Changing student attitudes through interaction: Findings from an interprofessional workshop. Journal of Interprofessional Care, v. 31, n. 4, p. 540-542, 2017. DOI: 10.1080/13561820.2017.1287165. Acesso em: 12 maio 2021.

GRANRUD, M. D. et al. Public health nurses' perceptions of interprofessional collaboration related to adolescents' mental health problems in secondary schools: A phenomenographic study. Journal of Clinical Nursing, v. 28, n. 15-16, p. 2899-2910, 2019. DOI: 10.1111/jocn.14881. Acesso em: 12 maio 2021.

HANSON, R. F. et al. Community-based learning collaboratives and participant reports of interprofessional collaboration, barriers to, and utilization of child trauma services.

Children and Youth Services Review, v. 94, p. 306-314, 2018. DOI:

10.1016/j.childyouth.2018.09.038. Acesso em: 12 maio 2021.

HAYWARD, L. et al. Interprofessional Collaboration Among Physical Therapy, SpeechLanguage Pathology, and Engineering Faculty and Students to Address Global Pediatric

Rehabilitation Needs: A Case Report. Journal of Physical Therapy Education, v. 30, n. 4, p. 24-34, 2016. DOI: 10.1097/00001416-201630040-00005. Acesso em: 12 maio 2021.

HERATH, C. et al. A comparative study of interprofessional education in global health care: A systematic review. Medicine, v. 96, n. 38, e7336, 2017. DOI:

10.1097/MD.0000000000007336. Acesso em: 12 maio 2021. 
HESJEDAL, E.; HETLAND, H.; IVERSEN, A. C. Interprofessional collaboration: Selfreported successful collaboration by teachers and social workers in multidisciplinary teams. Child and Family Social Work, v. 20, n. 4, p. 437-445, 2015. DOI: 10.1111/cfs.12093. Acesso em: 12 maio 2021.

HOOD, R. et al. Collaborating across the threshold: The development of interprofessional expertise in child safeguarding. Journal of Interprofessional Care, v. 31, n. 6, p. 705-713, 2017. DOI: 10.1080/13561820.2017.1329199. Acesso em: 12 maio 2021.

KALICHMAN, A. O.; AYRES, J. R. C. M. Comprehensiveness and healthcare technologies: a narrative on conceptual contributions to the construction of the comprehensiveness principle in the Brazilian Unified National Health System. Cadernos de Saúde Pública, v. 32, n. 8, e00183415, 2016. DOI: 10.1590/0102-311X00183415. Acesso em: 12 maio 2021.

KIM. J. et al. Interprofessional global health competencies of South Korean health professional students: educational needs and strategies. BMC Medical Education, v. 19, n. 1, p. 429, 2019. DOI: 10.1186/s12909-019-1826-1. Acesso em: 12 maio 2021.

KORNAROS, K. et al. A hermeneutic study of integrating psychotherapist competence in postnatal child health care: Nurses' perspectives. BMC Nursing, v. 17, n. 1, p. 1-10, 2018. DOI: 10.1186/s12912-018-0311-1. Acesso em: 12 maio 2021.

LAM, W.; CHAN, E. A.; YEUNG, K. S. S. Implications for school nursing through interprofessional education and practice. Journal of Clinical Nursing, v. 22, n. 13-14, p. 1988-2001, 2013. DOI: 10.1111/jocn.12163. Acesso em: 12 maio 2021.

LANGHAM, G. W. et al. Healthy Kids: Impacting Children's Health in Rural Alabama. Online Journal of Rural Nursing and Health Care, v. 17, n. 1, p. 3-22, 2017. DOI: 10.14574/ojrnhc.v17i1.432. Acesso em: 12 maio 2021.

MAHLER, C. et al. Students' perception of interprofessional education in the bachelor programme "Interprofessional Health Care" in Heidelberg, Germany: an exploratory case study. BMC Medical Education, v. 18, n. 1, p. 19, 2018. DOI: 10.1186/s12909-018-11243. Acesso em: 12 maio 2021.

MARTINUSSEN, M. et al. Improving interprofessional collaboration in a community setting: Relationships with burnout, engagement and service quality. Journal of Interprofessional Care, v. 26, n. 3, p. 219-225, 2012. DOI: 10.3109/13561820.2011.647125. Acesso em: 12 maio 2021.

MARTINUSSEN, M. et al. Reorganisation of healthcare services for children and families: Improving collaboration, service quality, and worker well-being. Journal of Interprofessional Care, v. 31, n. 4, p. 487-496, 2017. DOI: 10.1080/13561820.2017.1316249. Acesso em: 12 maio 2021. 
MIGUEL, E. A. et al. Importance of interprofessional work for the Family Health Strategy and Pediatrics. Espaço para a Saúde - Revista de Saúde Pública do Paraná, v. 17, n. 2, p. 111-117, 2016. DOI: 10.22421/1517-7130.2016v17n2p111. Acesso em: 12 maio 2021.

MORGAN, S.; PULLON, S.; MCKINLAY, E. Observation of interprofessional collaborative practice in primary care teams: An integrative literature review. International Journal of Nursing Studies, v. 52, n. 7, p. 1217-1230, 2015. DOI: 10.1016/j.ijnurstu.2015.03.008. Acesso em: 12 maio 2021.

MORIN, C.; DESROSIERS, J.; GABOURY, I. Descriptive study of interprofessional collaboration between physicians and osteopaths for the pediatric population in Quebec, Canada. BMC Health Services Research, v. 17, n. 1, p. 1-8, 2017. DOI: 10.1186/s12913017-2717-y. Acesso em: 12 maio 2021.

MUZYK, A. J. et al. An Interprofessional Course on Substance Use Disorders for Health Professions Students. Academic Medicine: Journal of the Association of American Medical Colleges, v. 92, n. 12, p. 1704-1708, 2017. DOI: 10.1097/ACM.0000000000001766. Acesso em: 12 maio 2021.

NAGELKERK, J. et al. Patient safety culture transformation in a children's hospital: An interprofessional approach. Journal of Interprofessional Care, v. 28, n. 4, p. 358-364, 2014. DOI: 10.3109/13561820.2014.885935. Acesso em: 12 maio 2021.

ODEGARD, A.; STRYPE, J. Perceptions of interprofessional collaboration within child mental health care in Norway. Journal of Interprofessional Care, v. 23, n. 3, p. 286-296, 2009. DOI: 10.1080/13561820902739981. Acesso em: 12 maio 2021.

OGENCHUK, M.; SPURR, S.; BALLY, J. Caring For Kids Where They Live: Interprofessional collaboration in teaching and learning in school settings. Nurse Education in Practice, v. 14, n. 3, p. 293-298, 2013. DOI: 10.1016/j.nepr.2013.11.003. Acesso em: 12 maio 2021.

PEDUZZI, M. et al. Interprofessional education: training for healthcare professionals for teamwork focusing on users. Revista da Escola de Enfermagem da USP, v. 47, n. 4, p. 977-983, 2013. DOI: 10.1590/S0080-623420130000400029. Acesso em: 12 maio 2021.

PEDUZZI, M. The SUS is interprofessional. Interface - Comunicação, Saúde, Educação, v. 20, n. 56, p. 199-201, 2016. DOI: 10.1590/1807-57622015.0383. Acesso em: 12 maio 2021.

PETERS, M. D. J. et al. Chapter 11: Scoping Reviews (2020 version). In: AROMATARIS, E.; MUNN, Z. (Ed.). JBI Reviewer's Manual, JBI, 2020. DOI: 10.46658/JBIMES-20-12. Acesso em: 12 maio 2021. 
PHILLIPS, J. D.; WALSH, M. A. Teaming up in child welfare: The perspective of guardians ad litem on the components of interprofessional collaboration. Children and Youth Services Review, v. 96, p. 17-26, 2019. DOI: 10.1016/j.childyouth.2018.11.016. Acesso em: 12 maio 2021.

POLO, K. M.; CAHILL, S. M. Interprofessional Collaboration to Support Children with Diabetes. The Open Journal of Occupational Therapy, v. 5, n. 3, 2017. DOI:

10.15453/2168-6408.1338. Acesso em: 12 maio 2021.

PSAILA, K. et al. Interprofessional collaboration at transition of care: Perspectives of child and family health nurses and midwives. Journal of Clinical Nursing, v. 24, n. 1-2, p. 160172, 2014. DOI: 10.1111/jocn.12635. Acesso em: 12 maio 2021.

REISS, M.; GREENE, C. A.; FORD, J. D. Is it time to talk? Understanding specialty child mental healthcare providers' decisions to engage in interdisciplinary communication with pediatricians. Social Science and Medicine, v. 175, p. 66-71, 2017. DOI:

10.1016/j.socscimed.2016.12.036. Acesso em: 12 maio 2021.

REUTERSWARD, M.; HYLANDER, I. Shared responsibility: school nurses' experience of collaborating in school-based interprofessional teams. Scandinavian Journal of Caring Sciences, v. 31, n. 2, p. 253-262, 2017. DOI: 10.1111/scs.12337. Acesso em: 12 maio 2021.

SÆBJØRNSEN, S. E. N.; WILLUMSEN, E. Service user participation in interprofessional teams in child welfare in Norway: vulnerable adolescents' perceptions. Child and Family Social Work, v. 22, n. 1995, p. 43-53, 2015. DOI: 10.1111/cfs.12242. Acesso em: 12 maio 2021.

SAINI, B. et al. An interprofessional learning module on asthma health promotion. American journal of pharmaceutical education, v. 75, n. 2, p. 30, 2011. DOI: 10.5688/ajpe75230. Acesso em: 12 maio 2021.

SALM, T. et al. Interprofessional education internships in schools: Jump starting change. Journal of Interprofessional Care, v. 24, n. 3, p. 251-263, 2010. DOI: 10.1080/13561820903051469. Acesso em: 12 maio 2021.

SHIMMURA, K.; TADAKA, E. Development of an interprofessional collaboration competency scale for children with medical complexity. BMJ Open, v. 8, n. 6, p. 1-11, 2018. DOI: 10.1136/bmjopen-2017-019415. Acesso em: 12 maio 2021.

SILVA, F. A. M. DA; CASSIANI, S. H. DE B.; FREIRE FILHO, J. R. Interprofessional Health Education in the Region of the Americas. Revista Latino-Americana de Enfermagem, v. 26, e3013, 2018. DOI: 10.1590/1518-8345.0000.3013. Acesso em: 12 maio 2021.

SOLOMON, P.; RISDON, C. A process oriented approach to promoting collaborative practice: Incorporating complexity methods. Medical Teacher, v. 36, n. 9, p. 821-824, 2014. DOI: 10.3109/0142159X.2014.917285. Acesso em: 12 maio 2021. 
SOUZA, G. C. et al. Teamwork in nursing: restricted to nursing professionals or an interprofessional collaboration? Revista da Escola de Enfermagem da USP, v. 50, n. 4, p. 642-649, 2016. DOI: 10.1590/S0080-623420160000500015. Acesso em: 12 maio 2021.

STEWART, M. et al. An interprofessional approach to improving paediatric medication safety. BMC Medical Education, v. 10, n. 19, 2010. DOI: 10.1186/1472-6920-10-19. Acesso em: 12 maio 2021.

TATLA, S. K. et al. Implementing a collaborative coaching intervention for professionals providing care to children and their families: An exploratory study. Journal of Interprofessional Care, v. 31, n. 5, p. 604-612, 2017. DOI: 10.1080/13561820.2017.1336990. Acesso em: 12 maio 2021.

TEIXEIRA, M. R.; COUTO, M. C. V.; DELGADO, P. G. G. Primary care and collaborative care in children and adolescents psychosocial interventions: facilitators and barriers. Ciência \& Saúde Coletiva, v. 22, n. 6, p. 1933-1942, 2017. DOI: 10.1590/141381232017226.06892016. Acesso em: 12 maio 2021.

TVEIT, C.; BELEW, J.; NOBLE, C. Prewarming in a Pediatric Hospital: Process Improvement Through Interprofessional Collaboration. Journal of Perianesthesia Nursing, v. 30, n. 1, p. 33-38, 2015. DOI: 10.1016/j.jopan.2014.01.008. Acesso em: 12 maio 2021.

WIDMARK, C. et al. What do we think about them and what do they think about us? Social representations of interprofessional and interorganizational collaboration in the welfare sector. Journal of Interprofessional Care, v. 30, n. 1, p. 50-55, 2016. DOI: 10.3109/13561820.2015.1055716. Acesso em: 12 maio 2021.

WITT SHERMAN, D. et al. Illustrating and Analyzing the Processes of Interprofessional Collaboration: A Lesson Learned from Palliative Care in Deconstructing the Concept. Journal of palliative medicine, v. 20, n. 3, p. 227-234, 2017. DOI:10.1089/jpm.2016.0332. Acesso em: 12 maio 2021. 